\title{
¿Es posible llevar a cabo una alimentación vegana en edad pediátrica? Riesgos y beneficios
}

\author{
Judith Calpe Salvador \\ al374088@uji.es \\ Águeda Cervera Gasch \\ cerveraa@uji.es
}

\section{Resumen}

Introducción: Alrededor de los 6 meses de vida, la leche deja de ser suficiente para atender las necesidades nutricionales del bebé y se recomienda iniciar la alimentación complementaria. Actualmente, hay un incremento de familias que eligen iniciar esta alimentación complementaria a través de una dieta vegana, siendo popularmente considerada de alto riesgo. A continuación, se explican los posibles riesgos y beneficios de llevar a cabo este tipo de alimentación.

Método: Se realizó una revisión de la literatura acerca de la alimentación vegana en edad pediátrica utilizando diferentes bases de datos: Google Académico, PubMed, Lilacs y Cochrane Library. Se emplearon una serie de descriptores basados en el lenguaje libre, en el vocabulario Medical Subject Headings (MeSH) y en los Descriptores en Ciencias de la Salud (DeCS). Se incluyeron los artículos relacionados con el tema de estudio.

Resultados: En PubMed se obtuvieron 44 artículos y, tras aplicar los criterios de exclusión, se aceptaron 6 . En Cochrane library no se obtuvo ningún artículo. Por último, en Lilacs se identificaron 8 artículos, de los cuales solo 1 cumplía con los requisitos. En total, se han encontrado 7 artículos relacionados con el tema.

Conclusiones: Las dietas veganas correctamente planificadas son saludables y nutricionalmente adecuadas en edad pediátrica ya que proporcionan un correcto desarrollo en los bebés. Sin embargo, hay muy poca literatura sobre el tema que permita conocer ampliamente los riesgos y los beneficios de una dieta vegana para que el personal sanitario esté correctamente informado y dé consejos a las familias.

Palabras clave: alimentación complementaria, dieta vegana, dieta vegetariana, infancia y lactante. 


\begin{abstract}
Introduction: After the first six months of life, milk is not enough to supply the nutritional necessities and it is recommended to start the complementary feeding. Currently, there is an increase in the number of families who adopt a vegan complementary feeding, which is considered to be high-risk. The possible risks and benefits of carrying out this type of feeding are explained below.

Method: This study is based on a literature review about vegan diets in pediatric ages using these databases: Google Academic Search, PubMed, Lilacs and Cochrane Library. A series of descriptors on free language vocabulary from Medical Subject Headings (MeSH) and Health Sciences Descriptors (DeCS) were used. Articles related to the aim of the study were included.

Results: In PubMed, 44 articles were obtained, but only 6 were accepted after using the exclusion criteria. There were no articles obtained from Cochrane Library. Finally, 8 articles were identified in Lilacs of which only 1 met the requirements. In total, 7 articles related to the study have been found.

Discussion: Vegan diets, correctly scheduled, are healthy and nutritionally appropriate in pediatric ages since they provide the correct development of the infants. On the other hand, there is very little literature about the topic which would allow to know more about the risks and benefits of a vegan diet so that Health professionals are well informed and able to counsel families.
\end{abstract}

Keywords: Complementary feeding, vegan diet, vegetarian diet, childhood and infant.

\title{
Introducción
}

Actualmente, el número de personas que eligen llevar una dieta vegana está aumentando considerablemente y, con ello, el incremento de pacientes en edad pediátrica que también adoptan este tipo de alimentación (Martínez Biarge 2017). Esto es debido principalmente a que los padres o tutores suelen instaurar en sus hijos/as esta forma de alimentarse desde las primeras etapas de la vida.

Una dieta vegana es aquella que se basa únicamente en alimentos vegetales, es decir, se abstiene de todo alimento o derivado de origen animal, como carnes, pescados, huevos, productos lácteos, miel... (Le Roy y Díaz 2010). Según Lozano (2017), es incluso un estilo de vida que va más allá de la alimentación, ya que también abarca el mundo de la cosmética y la moda y busca alternativas que apuesten por unos productos $100 \%$ vegetales y sostenibles.

El objetivo de este estudio es conocer, a través de una revisión de la literatura, si la alimentación vegana es nutricionalmente adecuada en niños y niñas una vez se inicia la alimentación complementaria. Además, se pretende conocer si existen riesgos o beneficios en comparación con la alimentación tradicional omnívora, identificando los posibles déficits nutricionales y cómo solventarlos.

Según la Sociedad Europea de Gastroenterología, Hepatología y Nutrición Pediátrica (EPSGHAN), este tipo de alimentación para lactantes se considera de alto riesgo de déficits nutricionales que podrían aparecer sin una buena planificación. Por ello, se 
necesitan profesionales sanitarios bien formados para ser capaces de reconocer e informar a los padres sobre qué nutrientes se pueden ver más comprometidos y cómo solventarlo, así como de informar sobre sus beneficios (Fewtrell et al. 2017).

Por otro lado, expertos de la Sociedad Científica para la Nutrición Vegetariana (SSNV) examinaron la literatura disponible sobre nutrición vegana en mujeres embarazadas, bebés lactantes y niños con el fin de resumir las recomendaciones más relevantes y concluyeron que es una dieta rica en una gran variedad de alimentos: granos, legumbres (incluida la soja y sus derivados), verduras, frutas, nueces y semillas, grasas vegetales, hierbas y especias (Baroni et al. 2018)

En relación con los bebés lactantes, no hay evidencia que considere la lactancia materna perjudicial en hijos de madres veganas. La lactancia materna exclusiva es preferible que sea la primera elección para la alimentación del lactante independientemente del tipo de dieta que se siga, ya que la leche de las mujeres veganas que siguen este tipo de dieta bien planificada, incluida una fuente confiable de vitamina B12, proporciona una nutrición adecuada para sus bebés amamantados (Baroni et al. 2018).

En cuanto a la introducción de la alimentación complementaria (AC), se suele iniciar de la misma manera para lactantes veganos y omnívoros, ya que las necesidades nutricionales son las mismas. Solamente cabría destacar la diferencia que supone introducir nutrientes provenientes de la carne o del pescado o introducir aquellos de origen vegetal y los riesgos que eso implica. Estudios recientes explican el posible déficit de nutrientes como ácidos grasos omega 3 , hierro, calcio, zinc, yodo, vitamina $D$, vitamina $B 12$, riboflavina y selenio, que son nutrientes implicados especialmente en funciones vitales como el crecimiento y desarrollo. Sin embargo, pueden ser aportados a través de una alimentación bien planificada, a excepción de la vitamina B12 que precisará ser suplementada (Baroni et al. 2018). En cambio, por otro lado, favorece una mayor ingesta de vitamina $\mathrm{C}$, folato, ácidos grasos omega 6 , fibra, potasio, vitamina $\mathrm{E}$, carotenoides y flavonoides.

En resumen, se puede llevar a cabo un aporte de nutrientes óptimo a partir de una dieta vegana siempre que esté bien planificada. Para ello, es de gran importancia la formación en este ámbito de los profesionales sanitarios y poder asesorar correctamente a estos pacientes. Además, es necesario que se conozca que los posibles déficits nutricionales que puede conllevar la dieta vegana se pueden solventar a partir de una alimentación bien planificada, a excepción de la vitamina B12 que deberá suplementarse (Sanchis, Redondo y Codoñer 2016).

\section{Método}

Para realizar este estudio, se llevó a cabo una revisión integradora de la literatura para conocer la mejor evidencia disponible sobre riesgos y beneficios de la alimentación vegana en edad pediátrica.

Se emplearon una serie de descriptores para establecer los términos de investigación en lenguaje natural, en el vocabulario Medical Subject Headings (MeSH) de la Biblioteca Nacional de Estados Unidos y en los Descriptores en Ciencias de la Salud (DeCS) de la Biblioteca Virtual en Salud para obtener los resultados finales.

Las bases de datos, revistas científicas y metabuscadores utilizados fueron: Google Académico, PubMed, Lilacs y Cochrane Library. Además, para asegurar la continua 
recolección de datos, se crearon alertas semanales en las diferentes fuentes de información.

Los descriptores utilizados fueron: Complementary feeding, vegan diet, vegetarian diet, child e infant, los cuales se combinaron con los operadores booleanos AND y OR para elegir los artículos que más se adaptaban a los objetivos de la revisión. En la figura 1 se observan los descriptores utilizados.

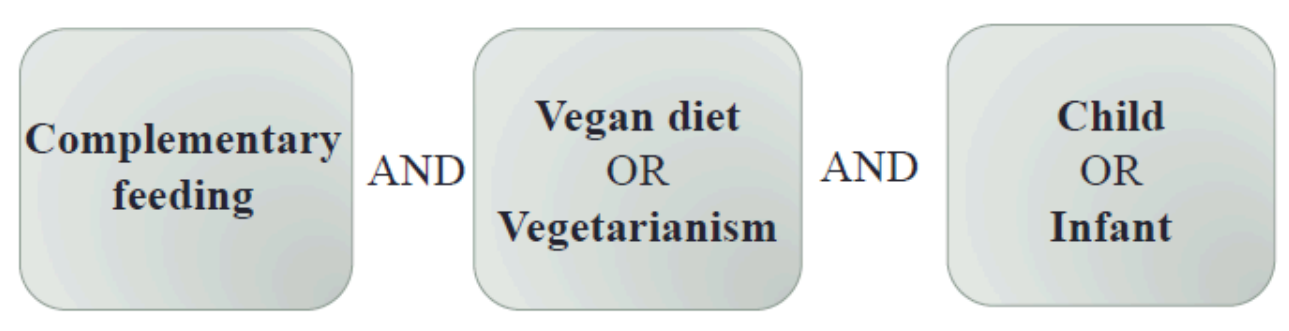

Figura 1. Estrategia de búsqueda.

Como criterios de inclusión se estableció: artículos relacionados con la alimentación vegana en edad pediátrica publicados después del año 2000, artículos con palabras clave en título o abstract y que la edad comprendida de los lactantes fuera entre 0 y 23 meses.

Por otro lado, se excluyeron los artículos que trataban sobre casos aislados y los relacionados solo con el embarazo.

\section{Resultados}

Tras la realización de la búsqueda anterior, se estableció la siguiente relación de artículos encontrados, descartados y seleccionados según los criterios de inclusión y exclusión establecidos y se identificaron un total de 52 artículos.

De estos, tras aplicar los criterios de exclusión, fueron aceptados solamente 7 artículos de interés para realizar la revisión bibliográfica, de los cuales 6 proceden de PubMed, 1 de Lilacs y ninguno de Cochrane Library (véase la tabla 1).

Además, los artículos encontrados en Google Académico coinciden con los de PubMed.

Tabla 1

Resultados de la búsqueda

Base de datos Número de artículos Artículos relacionados con el encontrados tema de estudio

\begin{tabular}{ccc}
\hline PubMed & 44 & 6 \\
\hline $\begin{array}{c}\text { Cochrane } \\
\text { Library }\end{array}$ & - & - \\
\hline Lilacs & 8 & 1 \\
\hline TOTAL & 52 & $\mathbf{7}$
\end{tabular}


En la figura 2 se muestra el diagrama de flujo donde aparece cómo se ha realizado la selección de los artículos que se incluirán en la revisión integradora.

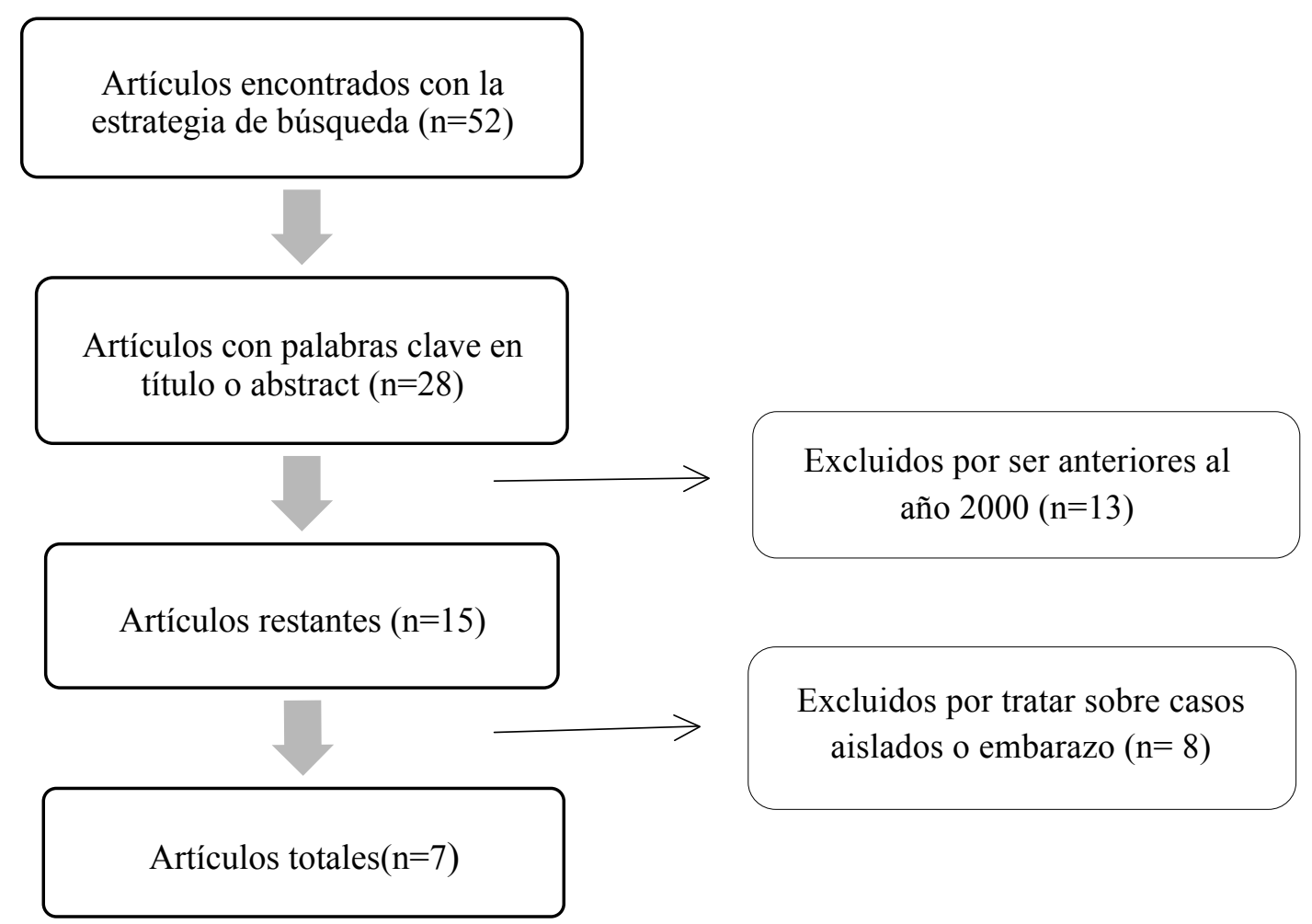

Figura 2. Diagrama de flujo para la selección de artículos.

Se obtuvo un número muy bajo de artículos relacionados con el tema a tratar, debido a que la mayoría hablaban de la alimentación vegana durante el embarazo o trataban sobre casos aislados, los cuales no tenían ninguna relevancia para este estudio.

Para facilitar la lectura de los artículos se han clasificado en la siguiente tabla (véase la tabla 2)

Tabla 2

Resumen de los artículos

\begin{tabular}{|c|c|c|c|c|}
\hline Autor & Título & Año & Revista & Línea de investigación \\
\hline Agostoni et al. & $\begin{array}{l}\text { Complementary } \\
\text { feeding: a commentary } \\
\text { by the ESPGHAN } \\
\text { Committee on Nutrition. }\end{array}$ & 2008 & $\begin{array}{l}\text { Journal of Pediatric } \\
\text { Gastroenterology } \\
\text { and Nutrition. }\end{array}$ & $\begin{array}{lr}\text { No recomiendan la } & \text { la } \\
\text { alimentación vegana } & \text { en } \\
\text { bebés y niños pequeños. } & \end{array}$ \\
\hline Baroni et al. & $\begin{array}{l}\text { Vegan Nutrition for } \\
\text { Mothers and Children: } \\
\text { Practical Tools for } \\
\text { Healthcare Providers. }\end{array}$ & 2018 & MDPI - Nutrients & $\begin{array}{l}\text { Recomendaciones de un } \\
\text { panel de expertos sobre los } \\
\text { alimentos necesarios para } \\
\text { una adecuada alimentación } \\
\text { vegana. }\end{array}$ \\
\hline
\end{tabular}




\begin{tabular}{|c|c|c|c|c|}
\hline Autor & Título & Año & Revista & Línea de investigación \\
\hline Fewtrell et al. & $\begin{array}{l}\text { Complementary } \\
\text { Feeding: A Position } \\
\text { Paper by the European } \\
\text { Society for Paediatric } \\
\text { Gastroenterology, and } \\
\text { Hepatology, } \\
\text { Nutrition (ESPGHAN) } \\
\text { Committee on Nutrition. }\end{array}$ & 2017 & $\begin{array}{l}\text { Journal of Pediatric } \\
\text { Gastroenterology } \\
\text { and Nutrition. }\end{array}$ & $\begin{array}{l}\text { Se pueden ofrecer dietas } \\
\text { veganas en niños pero bajo } \\
\text { supervisión para planificar } \\
\text { correctamente } \\
\text { alimentación. }\end{array}$ \\
\hline $\begin{array}{l}\text { American } \\
\text { Dietetic } \\
\text { Association } \\
\text { and Dietitians } \\
\text { of Canada }\end{array}$ & 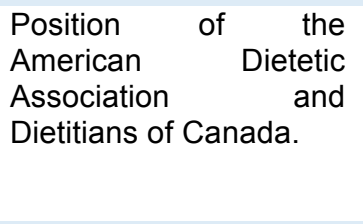 & 2003 & $\begin{array}{lrr}\text { Journal of } & \text { the } \\
\text { Academy } & & \text { of } \\
\text { Nutrition } & & \text { and } \\
\text { Dietetics } & & \end{array}$ & $\begin{array}{l}\text { Consideraciones nutricionales } \\
\text { para veganos de proteínas, } \\
\text { hierro, zinc, } \begin{array}{r}\text { calcio, } \\
\text { vitamina } D,\end{array} \\
\text { vitamina B12, vitavina, } \\
\text { ácidos grasos y yodo. }\end{array}$ \\
\hline $\begin{array}{l}\text { Reed } \\
\text { Messina }\end{array}$ & $\begin{array}{l}\text { Considerations in } \\
\text { planning vegan diets. }\end{array}$ & 2001 & $\begin{array}{lrr}\text { Journal of } & \text { the } \\
\text { Academy } & & \text { of } \\
\text { Nutrition } & & \text { and } \\
\text { Dietetics } & & \end{array}$ & $\begin{array}{l}\text { Afirman que las dietas } \\
\text { veganas pueden promover el } \\
\text { crecimiento normal de los } \\
\text { bebés siempre que se } \\
\text { proporcionen alimentos } \\
\text { utilizando pautas como las de } \\
\text { este artículo. }\end{array}$ \\
\hline $\begin{array}{l}\text { Van Winckel, } \\
\text { Vande Velde, } \\
\text { De Bruyne y } \\
\text { Van Biervliet }\end{array}$ & $\begin{array}{l}\text { Clinical practice: } \\
\text { vegetarian infant and } \\
\text { child nutrition. }\end{array}$ & 2011 & Eur J Pediatr & $\begin{array}{l}\text { Una dieta vegana debe ser } \\
\text { complementada al menos con } \\
\text { vitamina B12 y con especial } \\
\text { atención al calcio y zinc y } \\
\text { alimentos densos en energía } \\
\text { que contengan suficiente } \\
\text { proteína de alta calidad para } \\
\text { los niños pequeños. }\end{array}$ \\
\hline $\begin{array}{l}\text { Rojas, } \\
\text { Figueras } \\
\text { Durán }\end{array}$ & $\begin{array}{l}\text { Ventajas y desventajas } \\
\text { nutricionales de ser } \\
\text { vegano o vegetariano. }\end{array}$ & 2017 & $\begin{array}{l}\text { Revista chilena de } \\
\text { nutrición }\end{array}$ & $\begin{array}{l}\text { Alimentación vegana a lo } \\
\text { largo del ciclo vital } \\
\text { considerando los riesgos y } \\
\text { beneficios y cómo solventar } \\
\text { los posibles déficits } \\
\text { nutricionales que pueden } \\
\text { aparecer. }\end{array}$ \\
\hline
\end{tabular}

\section{Discusión y conclusiones}

Tras realizar la revisión integradora, se ha encontrado suficiente evidencia para afirmar que este tipo de dieta se considera, siempre que esté bien planificada, apropiada para los niños y las niñas de todas las edades, así como para todas las etapas del ciclo vital. Sin embargo, este tipo de alimentación ha sido identificada a lo largo de los años como deficitaria por la posible carencia de algunos nutrientes que pueden suponer graves consecuencias para el desarrollo y el crecimiento.

Algunos estudios, como los dirigidos por Fewtrell et al. (2017) y Baroni et al. (2018), coinciden en que este tipo de alimentación es de alto riesgo para los lactantes por los posibles déficits nutricionales que pueden aparecer siempre que no esté bien planificada. Esto es debido a que hoy en día los casos aislados de malnutrición en niños veganos se han relacionado casi exclusivamente con la inadecuación de la dieta ofrecida al bebé, ya que una dieta vegana organizada y que sigue todos los criterios que la definen como adecuada está completa.

Estos criterios para considerar la dieta adecuada, según Baroni et al. (2018), son el consumo de una variedad de alimentos vegetales durante todo el día sin excluir ningún grupo de alimentos de origen vegetal y centrar la atención en los nutrientes potencialmente críticos, es decir, aquellos que no pueden proporcionarse 
automáticamente por la variedad de los alimentos consumidos. Según este estudio, los nutrientes críticos incluyen proteínas, ácidos grasos omega-3, hierro, zinc, yodo, calcio, vitamina D y B12.

Además, la investigación de Baroni et al. (2018) no solo expone que bien planificada puede evitar la carencia de los nutrientes más comprometidos, sino que, además, defiende que es rica en una gran variedad de alimentos como legumbres (incluida la soja y sus derivados), verduras, frutas, nueces y semillas, grasas vegetales, hierbas y especias, lo que favorece una mayor ingesta de folato, fibra, potasio, vitamina $C$ y vitamina $E$, entre otros. Además, comentan los beneficios de llevar a cabo este tipo de alimentación, tales como tener un menor riesgo de desarrollar obesidad, estar menos expuestos a los antibióticos veterinarios que se encuentran en los alimentos derivados de animales y mostrar un perfil de adipocinas antiinflamatorias más favorable. Por otra parte, también explican cuáles son estos nutrientes más comprometidos y cómo llegar a una ingesta diaria adecuada, tales como proteínas, ácidos grasos omega-3, hierro, zinc, yodo, calcio, vitamina D y vitamina B12.

Otros estudios como el realizado por la Asociación Dietética Americana y Dietistas de Canadá (2003) y por Rojas, Figueras y Durán (2017) también coinciden en los posibles nutrientes deficitarios y detallan cómo llegar a su consumo correcto mediante los diferentes alimentos vegetales que encontramos en esta dieta.

Por tanto, los estudios realizados por Baroni et al. (2018), Fewtrell et al. (2017), la Asociación Dietética Americana y Dietistas de Canadá (2003) y Rojas, Figueras y Durán (2017) coinciden en los nutrientes que presentan más riesgo y a los que debemos prestar especial atención para evitar su deficiencia. Además, los tres últimos estudios mencionados detallan explícitamente cómo evitar la carencia de estos nutrientes.

Por otra parte, según las líneas de investigación de Agostoni et al. (2008), se recomienda evitar las dietas veganas en bebés y niños pequeños. En su investigación detallan que se han estudiado casos de bebés que han recibido una alimentación macrobiótica y han desarrollado enfermedades tales como retraso en el crecimiento, pérdida de grasa y músculos y un desarrollo psicomotor más lento, lo que establece la estricta necesidad de estar bajo supervisión médica o dietética apropiada. Sin embargo, se detallan las consecuencias de una dieta macrobiótica, no vegana; por lo que, a pesar de rechazar las dietas veganas, no se argumenta el por qué.

Como se ha comentado anteriormente, es una dieta que, bien planificada, no tiene por qué tener ninguna deficiencia, pero hay una excepción: la vitamina B12. Fewtrell et al. (2017) y la Asociación Dietética Americana y Dietistas de Canadá (2003) coinciden en que es necesaria la suplementación de vitamina B12 en bebés que no ingieren productos de origen animal en sus dietas, pero no detallan en qué momento debería iniciarse esta suplementación. Por otro lado, Van Winckel, Vande Velde, De Bruyne y Van Biervliet (2011) y Baroni et al. (2018) explican que es necesaria la suplementación de vitamina B12 una vez introducida la alimentación complementaria, es decir, alrededor de los 6 meses de edad. Sin embargo, se ha encontrado información en el estudio llevado a cabo por Rojas et al. (2017) que detalla que, durante la lactancia, si la madre no incluye en su alimentación ninguna fuente de vitamina B12 fiable (productos de origen animal o suplementos de estos) de forma regular, el lactante necesitará suplemento de vitamina B12, ya que la leche materna podría tener esta carencia. Por ello, la suplementación de vitamina B12 podría ser anterior a los 6 meses detallados por Van Winckel, Vande Velde, De Bruyne y Van Biervliet (2011) y Baroni et al. (2018). 
No obstante, Reed y Messina (2001) recomiendan, además de alimentos fortificados en vitamina B12, suplementos de calcio, zinc y vitamina D. Pero, al tratarse de un artículo previo a los mencionados anteriormente y cuya información no se ha vuelto a encontrar en ninguno de los artículos posteriores, se considera información incierta.

En conclusión, la mayoría de los estudios coinciden en que adoptar una dieta vegana bien planificada al inicio de la alimentación complementaria puede ser adecuada para bebés, niños y niñas, ya que ofrece un desarrollo y un crecimiento correcto desde su nacimiento. Además, es imprescindible prestar especial atención a aquellos nutrientes que podrían ser deficitarios en la dieta, los cuales pueden ser ingeridos perfectamente con una dieta adecuada, excepto la vitamina B12. Esta vitamina únicamente se encuentra en productos de origen animal, por lo que es de obligada suplementación.

Por otro lado, el incremento de personas que deciden adoptar este tipo de alimentación está aumentando año tras año, lo que presenta la necesidad de tener personal sanitario bien formado capaz de ofrecer consejos nutricionales correctos y adecuados a aquellas familias que deciden llevar esta alimentación para así evitar las complicaciones que podrían aparecer.

Por último, se considera una necesidad que se abran nuevas líneas de investigación, por una parte, debido a que existe poca literatura relacionada con el objeto de estudio y, por otra parte, para así despertar el interés de más profesionales sanitarios y que decidan aumentar sus conocimientos sobre el veganismo para asesorar con mayor evidencia y seguridad.

\section{Referencias bibliográficas}

Agostoni, Carlo, Tamas Decsi, Mary Fewtrell, Olivier Goulet, Sanja Kolacek, Berthold Koletzko, Kim Michaelsen, Luis Moreno, John Puntis, Jacques Rigo, Raanan Shamir, Hania Szajewska, Dominique Turck y Johannes van Goudoever. 2008. "Complementary Feeding: A Commentary by the ESPGHAN Committee on Nutrition». Journal of Pediatric Gastroenterology and Nutrition, 46(1): 99-110. Recuperado de: https://insights.ovid.com/crossref?an=00005176-20080100000021.

American Dietetic Association and Dietitians of Canada. «Position of the American Dietetic Association and Dietitians of Canada: Vegetarian diets». 2003. Journal of the Academy of Nutrition and Dietetics, 103(6): 748-765. Recuperado de: https://jandonline.org/article/S0002-8223(03)00294-3/fulltext

Baroni, Luciana, Silvia Goggi, Roseila Battaglino, Mario Berveglieri, Ilaria Fasan, Denise Filippin, Paul Griffith, Gianluca Rizzo, Carla Tomasini, Maria Alessandra Tosatti y Maurizio Antonio Battino. 2018. "Vegan Nutrition for Mothers and Children: Practical Tools for Healthcare Providers». Nutrients, 11(1): 5. Recuperado de: https://www.ncbi.nlm.nih.gov/pmc/articles/PMC6356233/\#

Fewtrell, Mary, Jiri Bronsky, Cristina Campoy, Magnus Domellöf, Nicholas Embleton, Natasha Fidler Mis, Iva Hojsak, Jessie M. Hulst, Flavia Indrio, Alexandre Lapillonne, y Christian Molgaard. 2017. «Complementary Feeding: A Position Paper by the European Society for Paediatric Gastroenterology, Hepatology, and Nutrition (ESPGHAN) Committee on Nutrition». Journal of Pediatric Gastroenterology and Nutrition, 64(1): 119-132. Recuperado de: https://journals.Iww.com/jpgn/fulltext/2017/01000/Complementary_Feeding_A _Position_Paper_by_the.21.aspx

Le Roy, Catalina y Ximena Díaz. 2010. «Dieta vegetariana en la edad pediátrica». Gastroenterología Latinoamericana, 21(1): 9-14. Recuperado de: http://gastrolat.org/DOI/PDF/10.0716/gastrolat2010n100002.pdf 
Lozano, Estefanía. 2017. «Veganismo: un estilo de vida sostenible». El País, 9 de marzo de 2017. Recuperado de:

https://elpais.com/elpais/2017/03/06/alterconsumismo/1488809957_092688.html

Martínez Biarge, Miriam. 2017. «Niños vegetarianos, ¿Niños sanos?». En curso de actualización pediátrica, ed. AEPap. Recuperado de:

https://www.aepap.org/sites/default/files/253-

268_ninos_vegetarianos_ninos_sanos.pdf

Reed, Ann y Virginia Messina. 2001. «Considerations in planning vegan diets». Journal of the Academy of Nutrition and Dietetics, 101(6): 670-677. Recuperado de: https://jandonline.org/article/S0002-8223(01)00169-9/fulltext

Rojas, Daniela, Francisca Figueras y Samuel Durán. 2017. «Ventajas y desventajas nutricionales de ser vegano o vegetariano». Revista chilena de nutrición, 44(3). Recuperado de: https://scielo.conicyt.cl/scielo.php?script=sci_arttext\&pid=S071775182017000300218

Sanchis-Chordà, J., Lucía Redondo y Pilar Codoñer. 2016. «Dieta vegana en la infancia: beneficios y riesgos». Revista española de pediatría, 72(5): 299-303. Recuperado

de: https://www.researchgate.net/publication/313239070_Dieta_vegana_en_la_infa ncia_beneficios_y_riesgos

Van Winckel, Myriam, Saskia Vande Velde, Ruth De Bruyne y Stephanie Van Biervliet. 2011 «Clinical practice: vegetarian infant and child nutrition». European Journal of Pediatrics, 170(12): 1489-1494. Recuperado de: https://www.ncbi.nlm.nih.gov/pubmed/21912895 